\title{
Grasping Action for Impaired Vision Action Using HC2D Barcode Technology
}

\author{
Puchong Subpratatsavee ${ }^{1}$, Suchai Tanaiadehawoot ${ }^{2}$ \\ Faculty of Science at Siracha, Kasetsart University Siracha Campus \\ 199 Sukumvit Rd. Siracha, Chonburi 20230 Thailand \\ puchong.sp@gmail.com ${ }^{1}$ fscisut@ku.ac.th ${ }^{2}$
}

\begin{abstract}
Nowadays, There are many people who have impaired vision. It is hard to do for weak-eyed man to sense a range because of their blurry vision. Therefore they often feel complication in grasping objects. It is useful to design and implement a system presenting the distance between a hand and an object for low vision people. In this paper, we propose a method to estimate the distance between a camera and an object surface on which a HC2D barcode is pasted. In this research, we assume the camera is worn on hand and the HC2D barcode with information embedded is pasted on the surface of object. The distance between the camera and the HC2D barcode, which is attached on the object, is estimated comparison the actual size of the HC2D barcode with the size of HC2D barcode that snap or capture from the camera.
\end{abstract}

Keywords: HC2D barcode, impaired vision, camera, grasping action

\section{Introduction}

According to the recent day, the lot of number of people with visual impairment in the world is low vision people. And people with visual impairment are elderly people who are old. It seems that the number of low vision people in the world increases much more. From these facts, developing a system, which supports low vision people's daily life, is necessary. Compared to the healthy people's vision (Fig. 1), low vision people's visibilities vary according to every individual symptom, such as blurry, crooked, and restricted visions. The visibility, of which an example is as shown in Fig. 2, might bring on tipping things and missing in grasping. And also, these difficulties could give low visions much stress. Indeed a lot of studies have been devoted with respect to support systems in reading characters, but there are few attempts to support in grasping things. In this paper, we propose a grasp action support system for visually impaired persons using HC2D barcodes [1] and a camera. This system can present the distance between the camera and the HC2D barcode, which is attached on the object surface by comparing the real size of HC2D barcode with the apparent size of HC2D barcode in the camera. In addition, the system can guide hands to the object's direction. The proposed system can be easily applied to support system present- 
ing the distance perception by wearing the camera on hand. The effectiveness of proposed system is verified through some experiments.

\section{Overviews}

In this section, the way to use the proposed system is explained. The purpose of system is to guide user's hand to the object on table safely by showing distance and direction information from the hand and an object. The user wears a small, lightweight camera on the hand. Cordless cameras are recommended so as to prevent the user from interfering in daily life. In using the system, captured images are transferred to processing equipment. And the distance and direction information are estimated when the system detects a HC2D barcode. It is useless for the visually impaired to show the distance and direction information in display because they cannot see anything. Therefore, this information are shown user by voice sound. If the captured HC2D barcode which is pasted on the object run off the center of camera coordinate at following the voice sound, voice sound guides the direction again to capture the HC2D barcode at the center. By repeating this operation, user's hand is guided to the object.

\section{$3 \quad$ Background and Related Works}

In this paper, we propose a new distance estimation method using a HC2D barcode and a camera. The reasons why we use HC2D barcode are shown as indicated below.

1. A special camera is unnecessary

2. It is easy to create HC2D barcode by using web sites.

3. The distance estimation can be quickly because HC2D barcodes can be read fast.

4. Extension of function is done easily by embedding some data in HC2D barcode such as the object's name.

In some research explain about distance estimation method using different-aperture images [2] using tilted lens optics. This method uses two different focus images. Comparing amount of the blur between these images performs distance estimation. However, this method needs to vary the aperture of camera at high speed in continuity. Therefore, it is hard to say this method is suitable for real-time processing. Some research show proposed method using a standing wave of audible sound. This method uses standing wave, which is generated by phase interference of transmitted and reflected waves. Using standing wave of audible sound [3], distance estimation is possible at the close distance. However, this method takes long time for calculating the distance. In addition, uses stereo images to estimate distance. Distance is estimated by the gap of corresponding areas. However, these methods do not use marker. It is not able to capture the target object. The purpose of this study is to guide a hand to object for visually impaired person. Therefore, it is hard to say this method is suitable for this study. As another approach, there is a method to use a compact compound-eye camera system: TOMBO [4]. However, TOMBO is special camera. In this study, a standard monocular camera is used from the viewpoint of availability and tractability. 


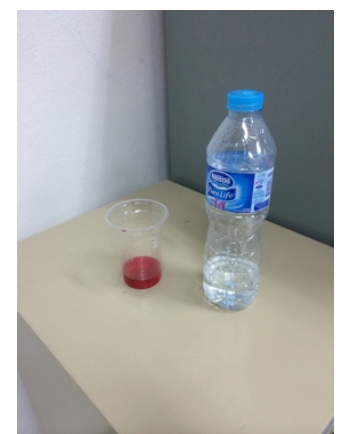

Fig. 1. Vision of healthy people

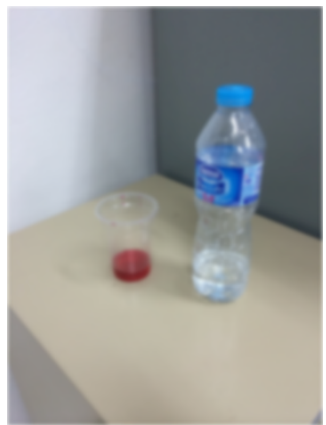

Fig. 2. Simulated low vision people's visibilities

\section{Proposed Method}

In this study, the distance between camera and the object is estimated by camera and HC2D barcode embedding the HC2D barcode's shape information. Let the camera focal length $f[m]$, distance between HC2D barcode and camera $z[m]$, size of HC2D barcode in the captured image a[pix], and real size of HC2D barcode $\mathrm{b}[\mathrm{m}]$. The relation of these symbols is shown in Fig. 3. As we can see, the distance between the camera and the HC2D barcode is calculated based two homologous triangles including camera focal length, the size of HC2D barcode in the captured image, and the real size of HC2D barcode. Processing flow of this study is shown as indicated below.

1. Get a captured image

2. Detect the region of HC2D barcode

3. Extract information of HC2D barcode

4. Estimate distance using the information of HC2D barcode

5. Show the distance the way to detect the region of HC2D barcode, way to estimate distance by using the information of HC2D barcode, and way to show the direction of object are explained in next section.

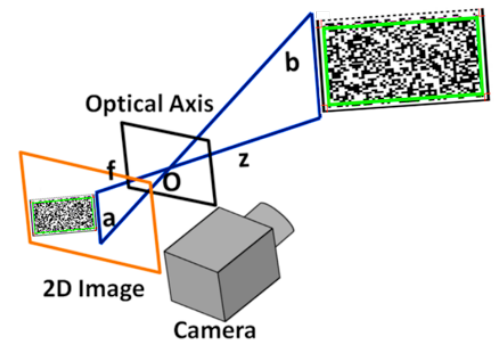

Fig. 3. Relationship of the camera and HC2D barcode 


\subsection{Detection of HC2D barcode}

In this section, the mathematical descriptions with respect to the distance estimation are explained. Suppose a camera coordinate is represented as (x; y; z) (Fig. 6), the coordinate is transformed into the image plane coordinate by perspective transformation as follows.

\subsection{Distance Estimation}

Three of four HC2D barcode's vertex has a distinctive mark called "finder pattern" (Fig. 4). HC2D barcode is distinguished by detecting these marks. To detect finder pattern, the following process are applied to the captured image.

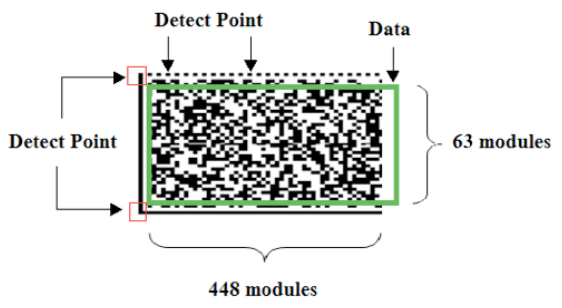

Fig. 4. HC2D barcode

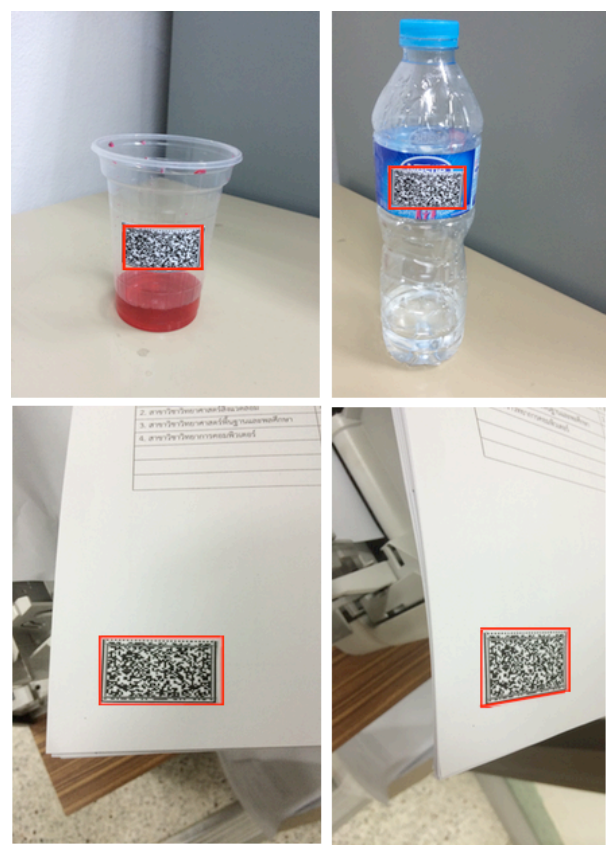

Fig. 5. Detection result of HC2D barcode

1. Binarize the captured image.

2. Extract the contour information.

3. Detect and save a square using the contour information. 
4. Check up the square whether it has Small Square in itself.

Assuming a realistic environment in which the systems used, the HC2D barcode is not always facing the front. Depending on the direction of HC2D barcode against for the camera, the square-shape code looks distorted such as a rectangle-shape one. Therefore, a certain range of aspect ratio is configured so that a little deviation is tolerated. At the end of these steps, a rectangle surrounded by a straight line connecting among the detected finder patterns is detected as the HC2D barcode. The result of detection of HC2D barcode is shown as Fig. 5. As shown in Fig. 5, if the HC2D barcode is taken on a skew in captured image, it is detected as HC2D barcode.

$$
(x, y, z) \rightarrow\left(f \frac{x}{z}, f \frac{y}{z}\right) .
$$

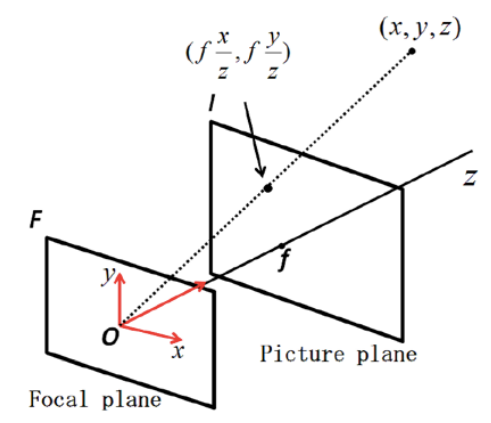

Fig. 6. Coordinate system

Indeed the above notation "(1)," is true in case of an ideal model, but the angle formed by $\mathrm{x}$-axis and $\mathrm{y}$-axis in the camera coordinate system does not definitely correspond to the angle $\theta$ formed by $u$-axis and v-axis in image plane. By considering the actual angle $\theta$, a coordinate $(x ; y ; z)$ is transformed to a point $(u ; v)$ in the image plane by the following equations:

$$
\begin{aligned}
& u=f k_{u} \cdot \frac{x+y \cot \theta}{z}+u_{0} \\
& v=f k_{v} \cdot \frac{y}{z \sin \theta}+v_{0}
\end{aligned}
$$

Where $f[\mathrm{~m}]$ is the focal length, $(u 0 ; v 0)$ is the coordinate of image center, $k_{u}[\mathrm{pix} / \mathrm{m}]$ is the inverse number of actual horizontal size of a pixel, and $k_{v}[\mathrm{pix} / \mathrm{m}]$ is the inverse number of actual vertical size of a pixel. Assume the HC2D barcode is a-pixel square in the image plane and $u 1$ and $u 2$ are vertex coordinates of HC2D barcode, $a$ [pix] is represented as the following equations:

$$
a=u_{1}-u_{2}
$$

Substituting the "(2)," to the "(4)," the following notation can be obtained:

$$
a=f k_{u} \frac{x_{1}-x_{2}}{z}
$$


Assume the real size of $\mathrm{HC} 2 \mathrm{D}$ barcode is $b[\mathrm{~m}]$, it is represented by the following equation:

$$
b=x_{1}-x_{2}
$$

Substituting the "(6)," to the "(5)," the following equation is obtained:

$$
z=f k_{u} \cdot \frac{b}{a}
$$

According to the "(7)," camera's focal length $f$ [m], distance between HC2D barcode and camera $z[\mathrm{~m}]$, size of HC2D barcode in the captured image $a[$ pix $]$ and real size of HC2D barcode $b[\mathrm{~m}]$ has proportional relation. From this point forward, the distance estimation is performed on the basis of the "(7)".

\subsection{Guide Process}

Two separated parts construct the guide method. One is a direction guide process to guide the user's hand to the object, and the other is distance guide process to announce the distance between the hand and the object.

\subsubsection{Direction Guide}

Captured image is separated 9 parts (Fig. 7). Direction guide is decided by detected position of HC2D barcode. There are 8 kinds of direction to guide the hand, such as "upper", "upper left", "upper right", "left", "right", "lower", "lower left", and "lower right". HC2D barcode's gravity center is calculated by detected HC2D barcode and direction of HC2D barcode is announced like "upper", "under left", by considering where the gravity point is found in separated parts. When the gravity point fit in the center of captured image, the hand is guided to the object after the distance between the hand and the object is announced.

\subsubsection{Distance Guide}

Distance between the camera and the object is calculated by "(7)," and user is informed the information of distance and direction by voice sound. When the HC2D barcode's gravity point fits in the center of captured image, the distance is read aloud for user. If the center of captured image does not fit the gravity point of HC2D barcode, voice sound announces the direction of the HC2D barcode again to fit the center point in gravity point. The system guides hand to the object by repeating this process. When the distance between hand and the object is short in under $5 \mathrm{~cm}$, voice sound saying that "please close your hand".

\section{$5 \quad$ Experimental Results}

\subsection{Measuring The Accuracy of Distance Estimation}

Distance estimation is conducted by HC2D barcode and camera using "(7)". In addition, the value of $f$ in " (7)" is calculated by camera calibration. Value of is calculated by (Fig. 7). In "(7)" substituting $a$ at $\mathrm{z}=0.05[\mathrm{~m}]$. In this experiment, the values of $\mathrm{f}$ 
and are used 838 and 0.686 , respectively. As described at section of "HC2D barcode detection", the camera does not always capture HC2D barcode from an anterior view in realistic environment. Within a captured image, there is a possibility of being reflected in the form where HC2D barcode was reflected aslant or was distorted. Therefore, distance estimations are attempted in some situations to evaluate the accuracy of estimated distance. The situation where distance estimation is performed is as follows. 1. When camera confront with HC2D barcode set as 0 degrees and measure distance which gradually changing the angle.

2. Estimate distance is performed at 3 patterns, which the HC2D barcode is attached on cylinder, prism and cone (Fig. 8).

The start of the estimate distance between HC2D barcode and camera is $0.03[\mathrm{~m}]$, and estimate distance interval is $0.005[\mathrm{~m}]$. If distance between camera and HC2D barcode will be less than $0.03[\mathrm{~m}], \mathrm{HC} 2 \mathrm{D}$ barcode cannot fit within the captured image.

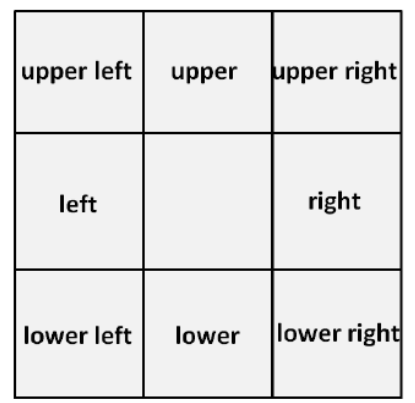

Fig. 7. Separate the captured image system

\subsection{Distance Estimation When HC2D barcode and A Camera Turn to The Front}

At first, distance estimation is conducted by camera and HC2D barcode, which is attached on flat plate surface. The estimated distance and true distance is summarized in graph. Axis of abscissas is true distance $D_{t}$ and axis of ordinate is error $E$. The error is calculated by estimated distance $D_{e}$ and true distance. If estimated distance is longer than true distance, the error is plotted with a positive value. By contraries, if estimated distance is shorter than true distance, the error is plotted with a negative value. The equation to calculate the error is shown as "(8)"

$$
E=\frac{D_{e}-D_{t}}{D_{t}} * 100
$$

Fig. 9 shows result of distance estimation when HC2D barcode and a camera turn to the front. The error was less than $5 \%$ and error was further reduced at close range. According to this result, our proposed method can estimate correct distance in this situation. 


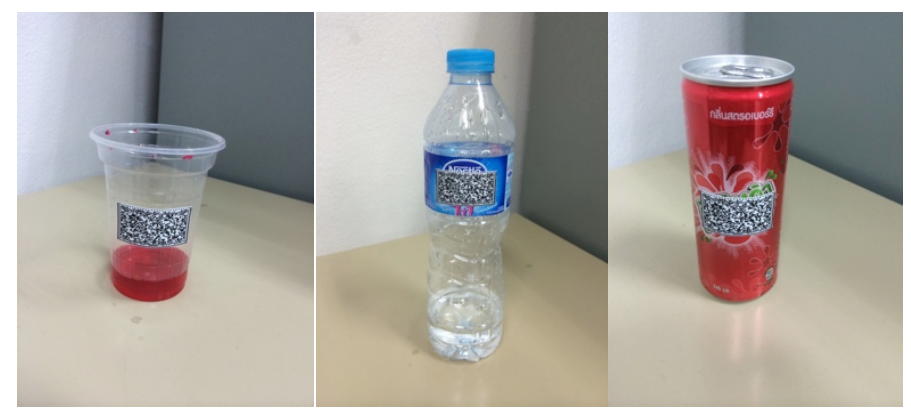

$\begin{array}{lll}\text { (a) Plastic cup } & \text { (b) Bottle } & \text { (c) Can }\end{array}$

Fig. 8. Attached place of HC2D barcode

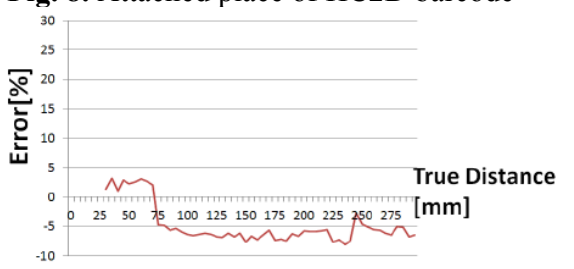

Fig. 9. Estimated error of distance between $\mathrm{HC} 2 \mathrm{D}$ barcode and a camera turn to the front

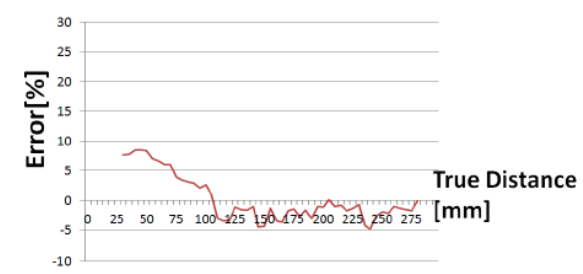

Fig. 10. Estimated error of distance between the HC2D barcode and the camera when the shooting angle is 15 degrees

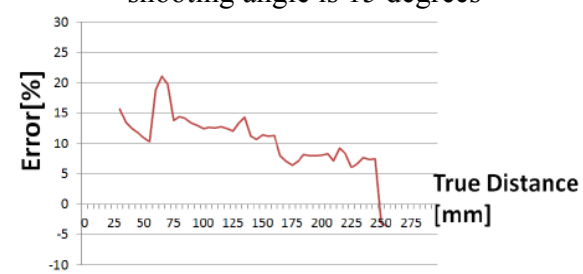

Fig. 11. Estimated error of distance between the HC2D barcode and the camera when the shooting angle is 30 degrees

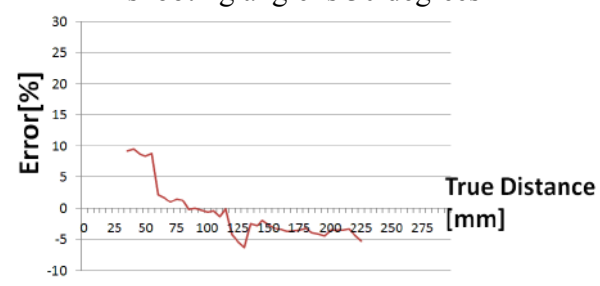

Fig. 12. Estimated error of distance between the HC2D barcode and the camera when the shooting angle is 45 degrees 


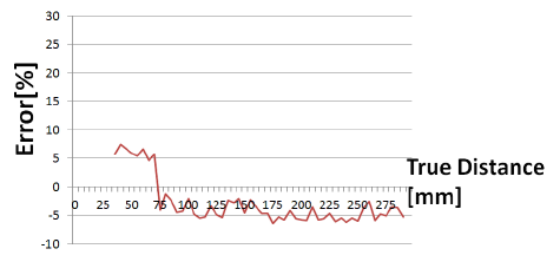

Fig. 13. Estimated error of distance between the HC2D barcode and the camera when the $\mathrm{HC} 2 \mathrm{D}$ barcode is pasted on cone

\subsection{Distance Estimation Whose Angle Changes Gradually}

When camera confront with HC2D barcode set as 0 degrees Distance estimation is performed whose angle shifts by every 15 degrees. When the angle exceeds 60 degrees, HC2D barcode did not recognize as HC2D barcode even if it is in the capture image. According to Figs. 10-12, in each angle, the accuracy of close range is lower than Fig.9. However, when the error of distance is replaced to real distance, it is only a several millimeters error. Therefore, this error does not affect action of grasp. The next experiment, HC2D barcode was attached on cylinder, prism and cone. We used tin can as cylinder, PET bottle as prism, and paper cup as cone. According to Figs. 13-15, the error of which HC2D barcode is attached on cylinder and cone, is similar at Fig. 9. However, the error of which HC2D barcode is attached on prism is huge. Furthermore, detection of HC2D barcode is difficult in this case.

\subsection{Assessment of Support System of Grasp Action}

Next, proposed system is evaluated by examinee by grasping the object using the system. First, examinees (sighted peoples) cut off the eyesight by taking a blindfold and put the camera on hand. After the blindfold, object, which the HC2D barcode has attached on, is set up on the desk (Fig. 16). The experiment whether the examinees can grasp the object or not is performed. The experiment environment is shown as indicated below. The number of examinees is 10 persons.

1. Examinees take a blindfold and do not wear the camera. They grasp the object at cluttered desk by only groping (Fig. 17): Valuation 1.

2. Examinees take a blindfold and wear the camera. They grasp the object using the system at desk put on the object only: Valuation 2.

3. Examinees take a blindfold and wear the camera. They grasp the object using the system at cluttered desk: Valuation 3.

\subsubsection{A. Valuation Method}

Examinees are had a questionnaire at 5 grade evaluation. The item of the questionnaire is shown as indicated below.

- Usability of the direction guide

- Usability of the distance guide

- Usability of this system 


\subsubsection{Result of Valuations}

The result of valuation 1 is shown as Table 1.7 of 10 blindfolded examinees could grasp the object at the cluttered desk by only groping. In this experiment, one examinee could grasp object, but he tipped other object on the desk. An average time the examinees grasp the object is 23 second. This experiment used only feeling of examinee's hand. Therefore, there are some cases that they tipped an object because of the difference between the examinee's imaged distance and the real distance. If it replaces to actual environment, it is dangerous for visually impaired persons to look for something only groping. If the tipped thing is hot drink, they get burned. We feed off the feedback from the examinees.

- It takes a long time to recognize that a touched object is target object or not because it is necessary that a touched object is confirmed carefully.

- It is dangerous that if there are some easily tipped things on the desk.

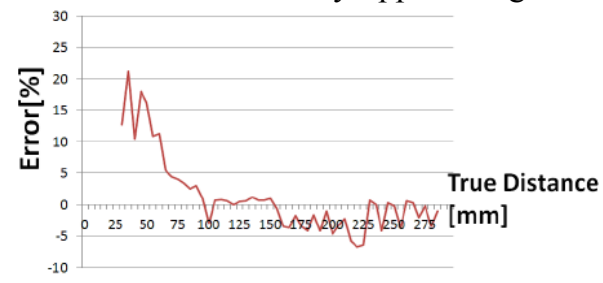

Fig. 14. Estimated error of distance between the HC2D barcode and the camera when the $\mathrm{HC} 2 \mathrm{D}$ barcode is pasted on cylinder

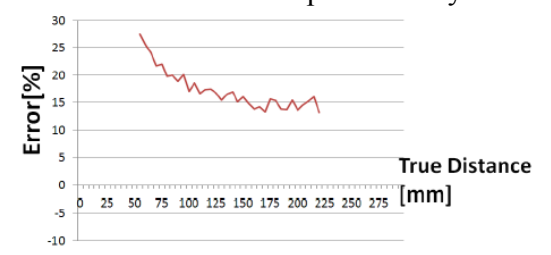

Fig. 15. Estimated error of distance between the HC2D barcode and the camera when the $\mathrm{HC} 2 \mathrm{D}$ barcode is pasted on prism

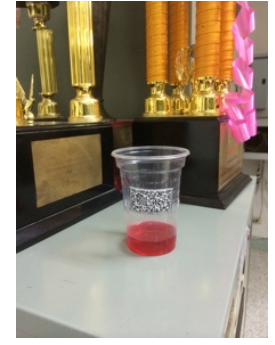

Fig. 16. Object, which the HC2D barcode has attached on

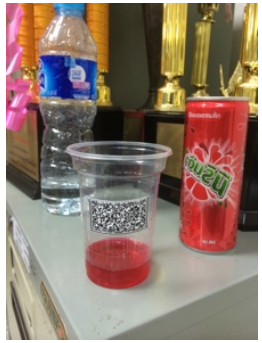

Fig. 17. The experiment consideringactual environment

Next, the result of valuation 2 is shown as Table 2. The value of "Usability of the direction guide", "Usability of the distance guide", and "Usability of this system" are shown at average point. In this experiment, there was only the object on the desk. Therefore, all examinees could grasp the object without the tipping. All items of usability value is over 3.5 . There is little variability among the examinees, but it seems to 
be good result. We feed off the feedback from the examinees. Sometimes, examinees feel difficulties that how long the camera moves. If the HC2D barcode's gravity point fits the center of captured image, it can grasp easily the object. It is necessary to get used to this system At last, the result of valuation 3 is shown as Table 3 and Table 4. The value of "Usability of the direction guide", "Usability of the distance guide", and "Usability of this system" are also shown at mean opinion score (MOS) [5]. As compared to the result of valuations 1, all examinees could grasp the object without the tipping. An average time is 4 second faster than the result of valuations 1 . Therefore, the information from the camera could guide examinee to the object. In addition, the evaluation of usability is better than the result of valuation 2 . It is because that the examinees get used to this system. These results show they alleviate their a burden on grasping action in daily life if users acquire proficiency in this system. We feed off the feedback from the examinees.

- When comparing the experiment 1 , it is easily to grasp the object by using this system's information.

- If the HC2D barcode out of the captured image, the system cannot inform the information of distance and direction.

Table 1. Result of practical experiments on using only groping

\begin{tabular}{lc}
\hline & A number of examinee \\
\hline The target was grasped, and nothing was tipped. & 6 \\
The target was grasped, but other object was tipped. & 1 \\
The target was tipped. & 3 \\
An average time the examinees grasp the object & 23 Second \\
\hline
\end{tabular}

Table 2. Mean option score at valuation 2. higher score is better. A number of examinee is 10 . (1: BAD, 2: POOR, 3: FAIR, 4: GOOD, 5: EXCELLENT.)

\begin{tabular}{cccc}
\hline $\begin{array}{c}\text { Average } \\
\text { Operate time }\end{array}$ & $\begin{array}{c}\text { Usability of the di- } \\
\text { rection guide }\end{array}$ & $\begin{array}{c}\text { Usability of the } \\
\text { distance guide }\end{array}$ & $\begin{array}{c}\text { Usability of } \\
\text { system }\end{array}$ \\
\hline 15.5 Second & $3.6 / 5$ & $3.5 / 5$ & $3.5 / 5$ \\
\hline
\end{tabular}

Table 3. Result of practical experiments on using the system

\begin{tabular}{cc}
\hline & A number of examinee \\
\hline The target was grasped, and nothing was tipped & 10 \\
The target was grasped, but other object was tipped & 0 \\
The target was tipped & 0 \\
An average time the examinees grasp the object & 19.3 Second \\
\end{tabular}


Table 4. Mean option score at valuation 3. higher score is better. A number of examinee is 10 . (1: BAD, 2: POOR, 3: FAIR, 4: GOOD, 5: EXCELLENT.)

\begin{tabular}{ccc}
\hline Usability of the direction guide & Usability of the distance guide & Usability of system \\
\hline $3.7 / 5$ & $3.3 / 5$ & $3.7 / 5$ \\
& & \\
\hline
\end{tabular}

\section{Conclusion}

In this paper, we propose distance estimation and guide system using HC2D barcode and a camera. This system can present distance between camera and HC2D barcode, which is attached on the object surface by comparing the real size of HC2D barcode with the size of HC2D barcode in the camera. In addition, the system can guide hands to the object's direction by capturing the HC2D barcode's position. By using this system, the visually impaired person's daily life ameliorates much more. In the experiment, we performed two prominent types of performs considering the actually environment. One is the measure of accuracy about distance estimation. We performed some experiments considering the actually environment and got a good result for this. Another is the validation of usability of guide system. The target object is set up on the desk with other object, and examinee grasped the target object using this system or does not. As a result, the value of valuation is over 3.5 point. All examinees could grasp the object by using this system, but $40 \%$ of examinees could not grasp the object by only using groping. Therefore, these results show this system's effectiveness. However, some examinees felt difficulties that how long they move their hand and where they move the hand if the camera drop off the HC2D barcode. To resolve this problem, the separation of captured image must become finer, and the direction will be announced more properly.

\section{References}

1. Subpratatsavee, P., and Kuacharoen, P.: An Implementation of a High Capacity 2D Barcode. In Advances in Information Technology, pp. 159-169. Springer Berlin Heidelberg (2012)

2. Pentland, A., et al.: A simple, real-time range camera. In: Computer Vision and Pattern Recognition, IEEE Computer Society Conference, pp.256-261 (1989)

3. Ohmata, N., Uebo, T., Nakasako, N., and Shinohara, T.: A trial on implementation of distance estimation method based on standing wave of audible sound. IEEJ Transactions on Electronics, Information and Systems, 129, 314-319 (2009)

4. Tanida, J., Kumagai, T., Yamada, K., Miyatake, S., Ishida, K., Morimoto, T., and Ichioka, Y.: Thin observation module by bound optics (TOMBO): concept and experimental verification. Applied Optics, 40(11), 1806-1813 (2001)

5. Viswanathan, M., \& Viswanathan, M.: Measuring speech quality for text-to-speech systems: development and assessment of a modified mean opinion score (MOS) scale. Computer Speech \& Language, 19(1), 55-83 (2005) 